\title{
OPTIMIZATION OF THE HOT-ELECTRON BOLOMETER AND A CASCADE QUASIPARTICLE AMPLIFIER FOR SPACE ASTRONOMY
}

\author{
Leonid Kuzmin $^{1}$
}

\section{INTRODUCTION}

Ultra low noise bolometers are required for space - based astronomical observations. Extremely sensitive detectors are necessary for a deep full-sky survey of distant extragalactic sources in the submillimeter-wave region corresponding to the extraterrestrial background spectrum minimum. A deep full-sky survey is the main goal of the Submillimetron project of the cryogenically cooled telescope on the International Space Station [1,2], project CIRCE (NASA) and other projects. Detection of faint sources involvves wide-band continuum observation using direct detectors (bolometers) that are not restricted by the quantum noise of indirect heterodyne receivers.

Theoretical estimations and preliminary experiments show that it is possible to realize the necessary sensitivity of $10^{-18}-10^{-19} \mathrm{~W} / \mathrm{Hz}^{1 / 2}$ with a novel concept of the antenna-coupled microbolometers $[3,4]$ at temperatures $\leq 0.1 \mathrm{~K}$ (Fig. 1). Additional advantages of such detectors are the possibility to operate with a wide range of background load, easy integration in arrays, and direct possibility of polarization measurements.

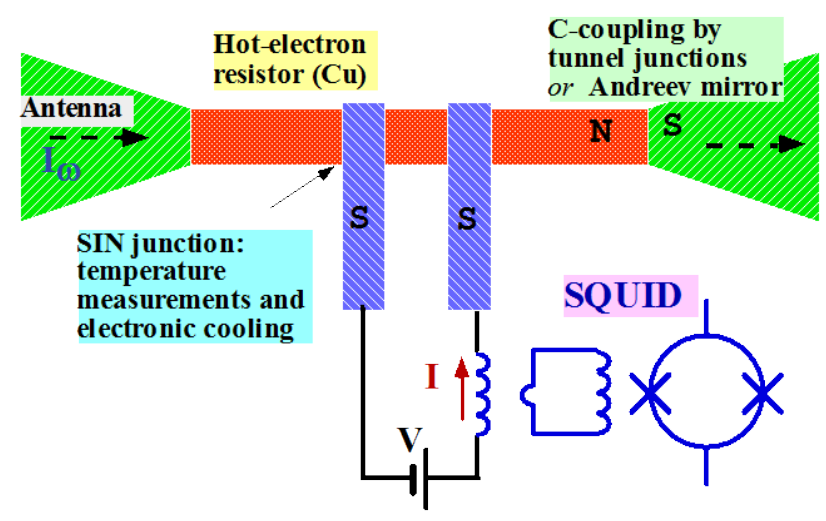

Fig. 1. Schematics of the normal metal hot-electron microbolometer (NHEB) with SIN tunnel junctions for temperature measurements in voltage biased mode with a SQUID readout system. Thermal isolation of absorber can be realized by SIN tunnel junctions (capacitive RF coupling) $[3,4]$ or by Andreev mirrors (direct RF coupling) $[5,6]$.

The normal metal hot-electron microbolometers (NHEB) with SIN tunnel junctions for temperature measurements was proposed in configuration with Andreev SN contacts for coupling to antenna [5-7]. However, due to limitation for frequencies less than superconducting gap and rather complicated structure this type of bolometer did not find further development. The antenna-coupled configuration $[3,4]$ has been proposed for the NHEB bolometer to improve sensitivity and high-frequency limits. The

\footnotetext{
${ }^{1}$ Leonid Kuzmin, Department of Physics, Chalmers University of Technology, S-41296 Göoteborg, Sweden. k kuzmin@fy.chalmers.se
} 
NHEB technology can be realized in two configurations: with tunnel junctions for thermal isolation and capacitive coupling to the antenna (NHEB-CC) [3,4] or with Andreev mirrors for thermal isolation (NHEB -A) [5-7], (Fig. 1). The NHEB-CC can be particularly useful for higher frequencies of operation not limited by the superconducting gap. The concept of "cold-electron" bolometer using direct electron cooling of the absorber by additional cooling SIN junctions [8] or temperature measuring SIN junctions [8] can considerably improve noise properties of the NHEB. It can particularly important in presence of a real background power load [9].

Another sensitive bolometer is a transition-edge sensor (TES) [10]working with additional heating of bolometer by dc bias power..

A very important question in our development strategy is whether the bolometer should operate at temperature of 100 or $300 \mathrm{mK}$. A temperature of $100 \mathrm{mK}$ is achievable by ADR, but $300 \mathrm{mK}$ is easier to achieve and cryogenic systems for this temperature are well-developed. Moreover, the electron cooling could be employed. We thus analyzed the system at $300 \mathrm{mK}$, trying to develop an "ideal model" of the detector. Experiments showed that direct electron cooling works perfectly at this temperature.

We have analyzed the concept of the optimal hot-electron bolometer in the presence of the final background power load $\left(P_{0}=10^{-13} \mathrm{~W}\right)$.

The optimal configuration of the bolometer proved to be the simplest configuration of the NHEB-CC with only 2 SIN tunnel junctions (Fig. 2). The tunnel junctions are used for simultaneous capacitive coupling to the antenna $[3,4]$ and for temperature measurements of the absorber. Besides that, the same tunnel junctions are used for direct electron cooling of absorber [8,9] and for thermal isolation of the absorber $[3,4]$.

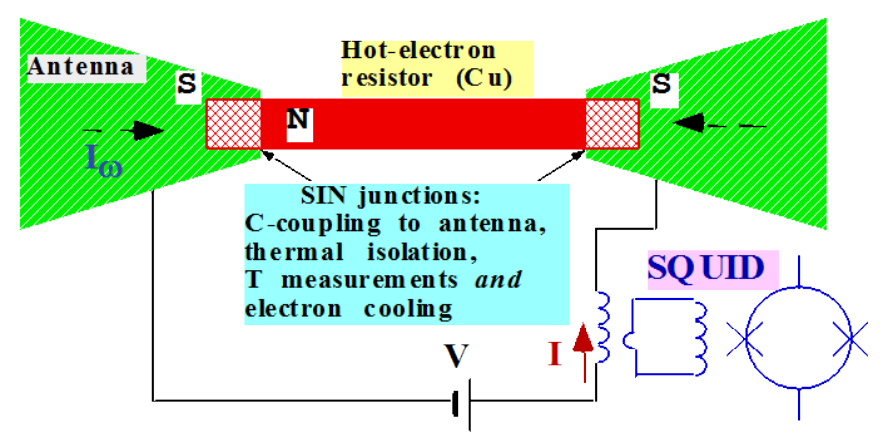

Fig. 2. a) Schematics of the optimal hot-electron microbolometer in the simplest configuration, the NHEBCC with two SIN tunnel junctions. The tunnel junctions are used for four functions: capacitive RF coupling to the antenna, thermal isolation of an absorber, temperature measurements and electron cooling of the absorber (Cold-Electron Bolometer).

Operation of the NHEB (Fig. 2) has been analyzed theoretically. The effect of correlation of the shot noise and the heat flow noise in the NIS junction has been discussed. Partial cancellation of the shot noise can be achieved in voltage-biased mode in contrast to the increased noise in the current-biased mode.

The analysis shows that a rather low NEP of better than $2 \times 10^{-19} \mathrm{~W} / \mathrm{Hz}^{1 / 2}$ determined by thermal fluctuation noise of a small absorber can be realized for typical volume of the absorber $0.05 \mu \mathrm{m}^{3}$. A SQUID can be used as a readout system in a voltage-biased mode. Experiments show that the NHEB technology makes it possible to produce a submillimeter wave receiver for space applications with a dc NEP of about $1.5 \times 10^{-18} \mathrm{~W} / \mathrm{Hz}^{1 / 2}$ at $300 \mathrm{mK}$ with SQUID amplifier.

\section{OPTIMIZATION OF THE BOLOMETER IN PRESENCE OF A BACKGROUND POWER LOAD}

\subsection{Model}

A heat balance equation for the normal metal strip $[8,9]$ is adopted in the following form: 
$c_{v} \Lambda \frac{d T_{e}}{d t}+\Sigma \Lambda\left(T_{e}^{5}-T_{p h}^{5}\right)+P\left(V, T_{e}, T_{S}\right)=P_{0}+\delta P$

Here $c_{v}=\gamma T_{e}$ is the specific heat capacity of the normal metal; $\Sigma \Lambda\left(T_{e}^{5}-T_{p h}^{5}\right)$ is the heat flow from the electron to the phonon subsystem in the normal metal, $\Sigma$ is a material constant, $\Lambda$ - a volume of the absorber, $T_{e}$ and $T_{p h}$ are, respectively, the electron and phonon temperatures; $T_{S}$ is the electron temperature of the superconductor electrode forming the SIN tunnel junction; $P_{0}$ is the background optical load of the bolometer, and $P(t)$ is the incoming rf power.

Here we assume that the NIS tunnel junctions are voltage-biased, and the current is measured by a SQUID. The sensitivity of the device is then characterized by the current responsivity $S_{I}$, which is the ratio of the current change detected by the SQUID and the change in the power load of the bolometer caused by a detected signal,

$S_{I}=\frac{\partial_{\omega}}{\partial P_{\omega}}=\frac{\partial / \partial T}{-i \omega C_{v} \Lambda+5 \Sigma \Lambda T_{e}^{4}+\frac{\partial P}{\partial T}}$.

The sum of the two heat conductances

$G_{e-p h}=5 \Sigma \Lambda T_{e}^{4}, \quad G_{S I N}=\frac{\partial P}{\partial T}$

stands in denominator.

Noise properties are characterized by the noise equivalent power (NEP), which is the sum of three different contributions, and is defined as follows:

$N E P_{\text {total }}^{2}=N E P_{e-p h}^{2}+N E P_{S I N}^{2}+\frac{\delta I^{2}}{S_{I}^{2}}$.

Here

$N E P_{e-p h}^{2}=10 k_{B} \Sigma \Lambda\left(T_{e}^{6}+T_{p h}^{6}\right)$

is the noise associated with electron-phonon interaction; $N E P^{2} S I N$ is the noise of the NIS tunnel junctions, and the last term $\delta \mathrm{I}^{2} / \mathrm{S}^{2} I$ is due to the finite sensitivity of the amplifier (SQUID) $\delta I$, which is expressed in $\mathrm{pA} / \mathrm{Hz}^{1 / 2}$ [9].

The noise of the SIN tunnel junctions, $N E P^{2} S I N$, has three components - shot noise $2 e I / S^{2}$, the fluctuations of the heat flow through the tunnel junctions and the correlation between these two processes

$N E P_{S I N}^{2}=\delta P_{\omega}^{2}-2 \frac{\delta P_{\omega} \delta I_{\omega}}{S_{I}}+\frac{\delta I_{\omega}^{2}}{S_{I}^{2}}$.

It is necessary to take into account the effect of the electron cooling of the metallic strip by the NIS tunnel junctions. For every chosen voltage we first solve the heat balance equation, find the electron temperature in the metallic strip, and only after that do we determine current responsivity and NEP. 


\subsection{NEP with background power load}

Our analysis of the effect of background power load on noise performance for different configurations of NHEB bolometers shows that the optimal configuration of the bolometer in presence of a realistic background power load is an NHEB with voltage-biased SIN tunnel junctions and current readout by a SQUID [9]. The volume of absorber is equal to $0.05 \mu \mathrm{m}^{3}$, which is typical for our experiments. The current noise of SQUID was equal to $50 \mathrm{fA} / \mathrm{Hz}^{1 / 2}$ in our simulations. The results are shown in Fig. 3 for two levels of microwave background power: $P_{0}=0$ and $0.1 \mathrm{pW}$. The latter figure is a realistic background power load $P_{0}$ for bandwidth $10 \%$ at frequencies $300-1000 \mathrm{GHz}$ for background temperature $T_{b g}=3 \mathrm{~K}$. The first curve without background load $\left(P_{0}=0\right)$ gives NEP $=2 \times 10^{-19} \mathrm{~W}$ for typical junction resistance (R) equal to $6 \mathrm{k} \Omega$. A considerable increase of the NEP to $8 \times 10^{-18} \mathrm{~W} / \mathrm{Hz}^{1 / 2}$ is obtained for $P_{0}=0.1 \mathrm{pW}$. The electron temperature also increases and reaches $230 \mathrm{mK}$. Decreasing $R$ to $0.5 \mathrm{k} \Omega$ improves the efficiency of the electronic cooling and returns the NEP to the acceptable level of $8 \times 10^{-19} \mathrm{~W} / \mathrm{Hz}^{1 / 2}$ and $T_{e}$ to the level of $100 \mathrm{mK}$. The NEP goal for the Submillimetron project is $10^{-18}$ $\mathrm{W} / \mathrm{Hz}^{1 / 2}[1]$.

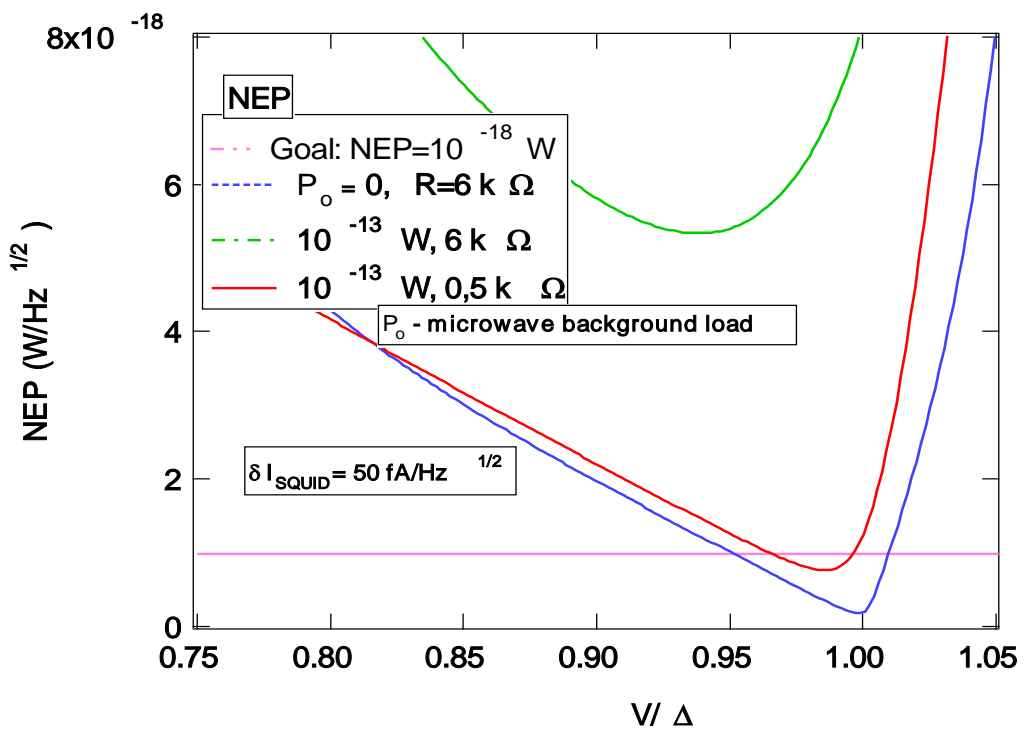

Fig. 3. a) NEP in presence of various background power loads and various efficiencies of direct electronic cooling for bath temperature $100 \mathrm{mK}$.

Overheating by the background power load is common for both types of sensors: NHEB and TES sensors using a small absorber. This overheating is dependent only on the volume of the absorber (or thermal conductance). Fortunately, the NHEB has a unique possibility of decreasing the overheating by direct electron cooling. By contrast, for normal operation of a TES, an additional dc power $P_{\text {bias }}=$ $\left(P_{\text {signal }}\right)_{\max }$ should be added to the background power, which would additionally increase the overheating.

\section{CONCEPT OF AN OPTIMAL BOLOMETER}

A very important question in our development strategy was whether the bolometer should operate at temperature of 100 or $300 \mathrm{mK}$. A temperature of $100 \mathrm{mK}$ is achievable by ADR, but $300 \mathrm{mK}$ is easier to achieve and cryogenic systems for this temperature are well-developed. Moreover, the electron cooling could be employed. We thus analyzed the system at $300 \mathrm{mK}$, trying to develop an "ideal model" 
of the detector. Experiments showed that direct electron cooling works perfectly at this temperature. Another option might be to use an intermediate temperature of $200 \mathrm{mK}$ using the double-stage ${ }^{3} \mathrm{He}$ sorption cooler recently developed by CEA, Grenoble.

We have analyzed the concept of the optimal hot-electron bolometer in the presence of the final background power load $\left(P_{0}=10^{-13} \mathrm{~W}\right)$ and for fixed parameters of the SQUID-amplifier $\left(10 \mathrm{fA} / \mathrm{Hz}^{1 / 2}\right)$. The optimal configuration of the bolometer in presence of the realistic background power load proved to be the simplest configuration, the NHEB-CC
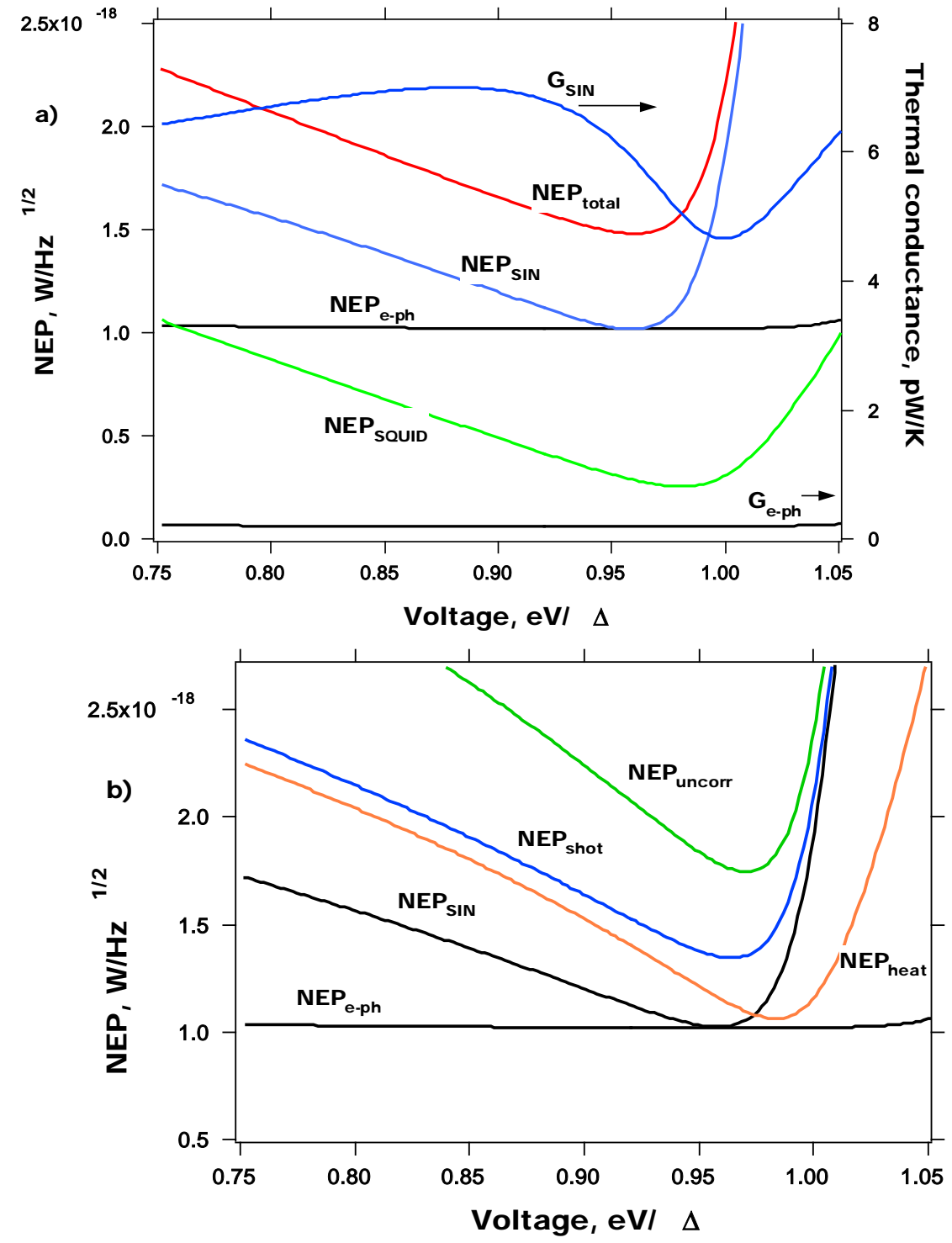

Fig. 4. a) NEP of an optimal bolometer in presence of the background power load $0.1 \mathrm{pW}$ for $\Lambda=0.001 \mu \mathrm{m}^{3}, \mathrm{R}=1$ $\mathrm{k} \Omega, \mathrm{S}_{\mathrm{SQUID}}=10 \mathrm{fA} / \mathrm{Hz}^{1 / 2}$, and for bath temperature $300 \mathrm{mK}$. b) $N E P$ SIN with partial cancellation of the shot noise $N E P_{\text {Shot }}$ and the heat flow noise $N E P_{\text {heat }}$ of SIN tunnel junction (6); $N E P_{\text {uncorr }}$ is shown for comparison. 
with two voltage-biased SIN tunnel junctions working in conditions of strong electron cooling and with current readout by a SQUID (Fig. 3). The optimal regime can be realized when thermal "cooling conductance" through the tunnel junctions predominates over "fundamental" electron-phonon conductance. In these circumstances, an NEP level of $10^{-18} \mathrm{~W} / \mathrm{Hz}^{1 / 2}$ at $300 \mathrm{mK}$ can be achieved. The typical values of NEP of an optimal bolometer in presence of the background power load $0.1 \mathrm{pW}, \mathrm{R}=1$ $\mathrm{k} \Omega, \mathrm{S}_{\mathrm{SQUID}}=10 \mathrm{fA} / \mathrm{Hz}^{1 / 2}$, and the optimal volume of absorber $\Lambda=0.0035 \mu \mathrm{m}^{3}$ are shown in Fig. 4a. For this volume, the total NEP (2) is determined by equal components of the noise of the SIN junction and the electron-phonon noise. Comparison of thermal conductance $\mathrm{G}_{\mathrm{e}-\mathrm{ph}}$ and $\mathrm{G}_{\mathrm{SIN}}$ shows that conductance through the SIN junctions predominates over electron-phonon conductance, resulting in practically full transferance of the incoming power to the readout amplifier. It is interesting to analyze the $\mathrm{NEP}_{\text {SIN }}$ (Fig. 4b) calculated in correspondence with equation (6) and uncorrelated noise $\mathrm{NEP}_{\text {uncorr }}$ including the first and third terms in equation (6).

The main components $\mathrm{NEP}_{\mathrm{SIN}}$, shot noise and heat flow noise (6), partly compensate each other due to mutual correlation and lead to reduction of $\mathrm{NEP}_{\text {SIN }}$ in comparison with $\mathrm{NEP}_{\text {uncorr }}$. This compensation is possible only in voltage-biased mode; in current-biased mode there would be opposite effect of increase of the noise to the level higher than uncorrelated noise $\mathrm{NEP}_{\text {uncorr }}[8]$.

The dependences of the NEP and thermal conductance $G$ of the bolometer on a volume of the absorber are shown in Fig. 5a. There is no optimal value of NEP for volume of absorber $\Lambda$ : if we continue to decrease $\Lambda$, the NEP will improve slightly, but really we have flattening of NEP at this level. The reason for the flattening is that we have achieved full transference of $P_{0}$ to the amplifier, so that the NEP $\mathrm{e}_{\text {-ph }}$ constitutes less that $50 \%$ of the total NEP. The critical point of optimal regime is a point of equality of $\mathrm{NEP}_{\text {SIN }}$ and $\mathrm{NEP}_{\mathrm{e}-\mathrm{ph}}$ shown by circle. The optimal regime is on the left from this point.

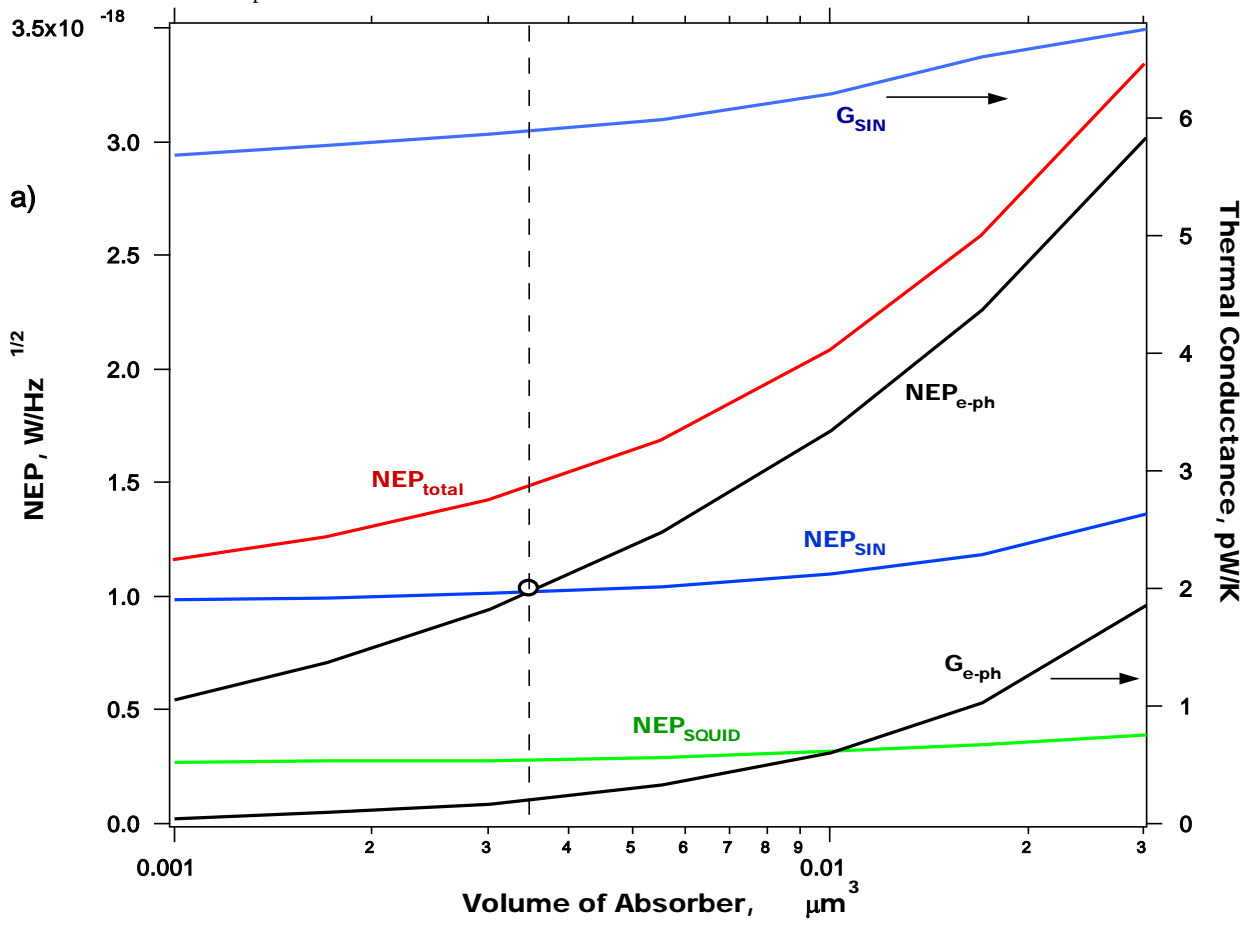




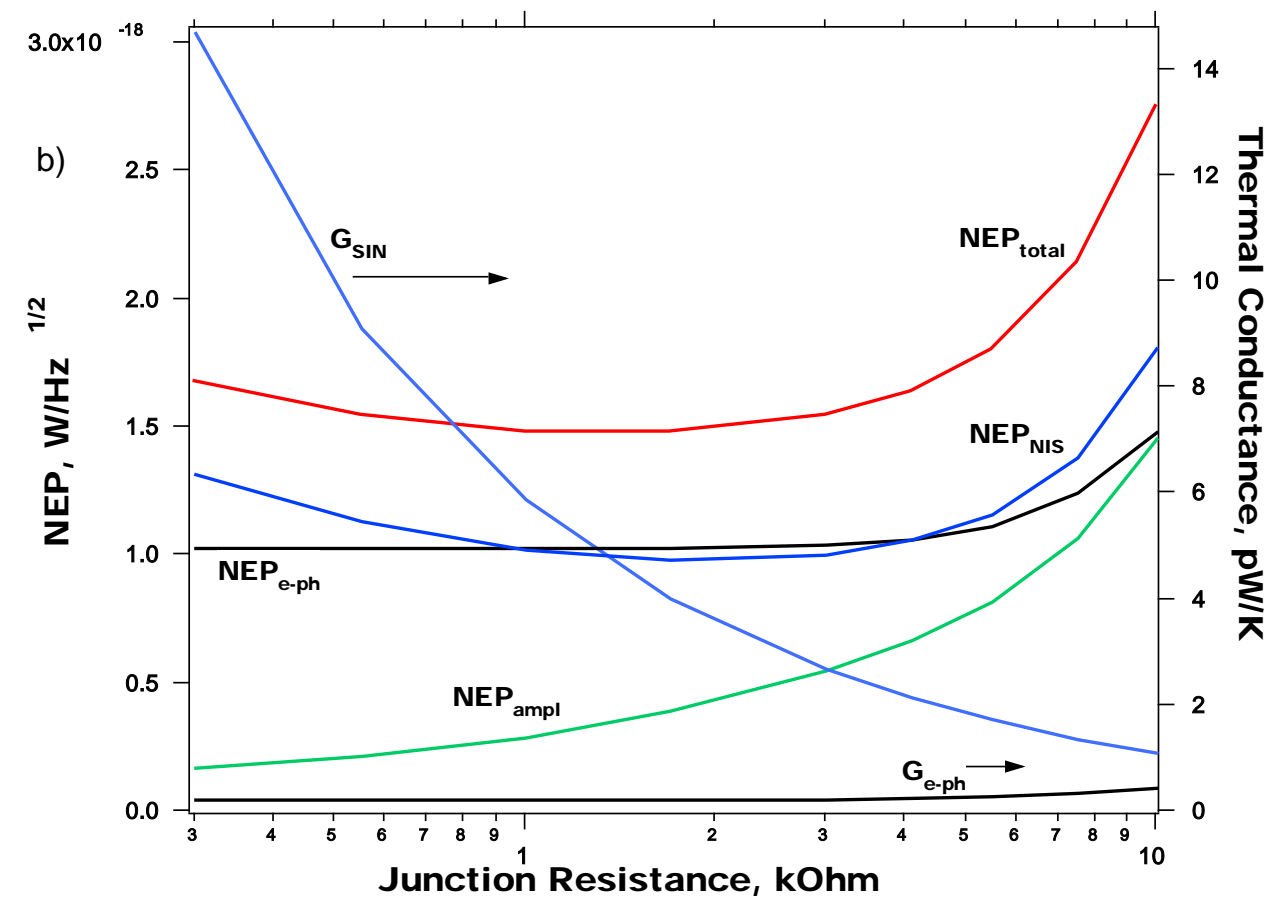

Fig. 5. a) NEP and thermal conductance of an optimal bolometer in dependence on volume of the absorber in presence of the background power load of $0.1 \mathrm{pW}$ for $\Lambda=0.001 \mu \mathrm{m}^{3}, \mathrm{R}=1 \mathrm{k} \Omega, \mathrm{S}_{\mathrm{SQUID}}=10 \mathrm{fA} / \mathrm{Hz}^{1 / 2}, \mathrm{~S}_{\mathrm{SQUID}}=10$ $\mathrm{fA} / \mathrm{Hz}^{1 / 2}$, and the bath temperature of $300 \mathrm{mK}$; b) the same values in dependence on resistance of the SIN tunnel junctions.

The dependences of the NEP and thermal conductance of the bolometer on the resistance of the SIN tunnel junctions $\mathrm{R}$ are shown in Fig. $5 \mathrm{~b}$. The optimum $\mathrm{R}$ value is around $1.5 \mathrm{k} \Omega$. For higher values of $\mathrm{R}$, the electron cooling is not so effective and responsivity is decreased increasing noise of SIN junction (6) and SQUID (4). Decrease of R increases the shot noise (reverse proportional to R) without any increase in responsivity because of saturation in transferring power.

It is interesting to note that equal values of $\mathrm{NEP}_{\text {SIN }}$ to $\mathrm{NEP}_{\mathrm{e}-\mathrm{ph}}$ correspond to considerably higher $\mathrm{G}_{\mathrm{SIN}}$ in comparison with $\mathrm{G}_{\mathrm{e}-\mathrm{ph}}$. That difference looks as shot noise of SIN junction is considerably "less noisy" than similar shot noise of phonons in electron-phonon system. In principle, the both type of noise are of the same order if we have the same temperature of the source of noise. Independently on small heat conductance $\mathrm{G}_{\mathrm{e}-\mathrm{ph}}(3)$ for the case of strong electron cooling, the relatively flat level of $\mathrm{NEP}_{\text {e-ph }}$ is determined by the second term of phonon noise in Eq.(5) due to fixed bath temperature. As a common result of optimal regime of bolometer, the intensive electron cooling helps to remove all background power load from the absorber without considerable increase of noise due to a low electron temperature of absorber as a source of the shot noise.

It gives great advantage and good perspectives of system with SIN tunnel junctions in contrast to a transition-edge sensor where all incoming power would overheat an absorber increasing the electron temperature to organize heat flow through the only available mechanism of electron-phonon cooling. Additional dc heating would only increase the electron temperature and the noise.

\section{CASCADE QUASIPARTICLE AMPLIFIER}


Effective electron cooling is very important for realization of good noise properties of the NHEB bolometer. The diagram in Fig. 6 shows the process of electron cooling with removing hot electrons from absorber $\mathrm{N}_{1}$ and creating hot quasiparticles in superconductor $\mathrm{S}_{1}$. After that, the quasiparticles are not needed for the measurement current because the current will be supported by Cooper pair in superconductor. To avoid back heating, the quasiparticles should be trapped by normal metal as soon as possible (see, for example, ref [9]). Each quasiparticle releases high energy $\Delta$ in the trap in contrast to removed energy from the absorber of the order of kT with very small efficiency of electron cooling. It is very attractive not throw away these quasiparticles but use their energy for amplification of measurement current.

Several attempts were made before to use a trap for multipliers and transistors [11-13]. However, realization of these devices has not been done yet. Due to our analysis, the main reason is saturation of the devices by background power load (overheating) due to high released energy of quasiparticles equal $\Delta$. The coefficient of amplification for small signal is considerably degraded due to this reason.
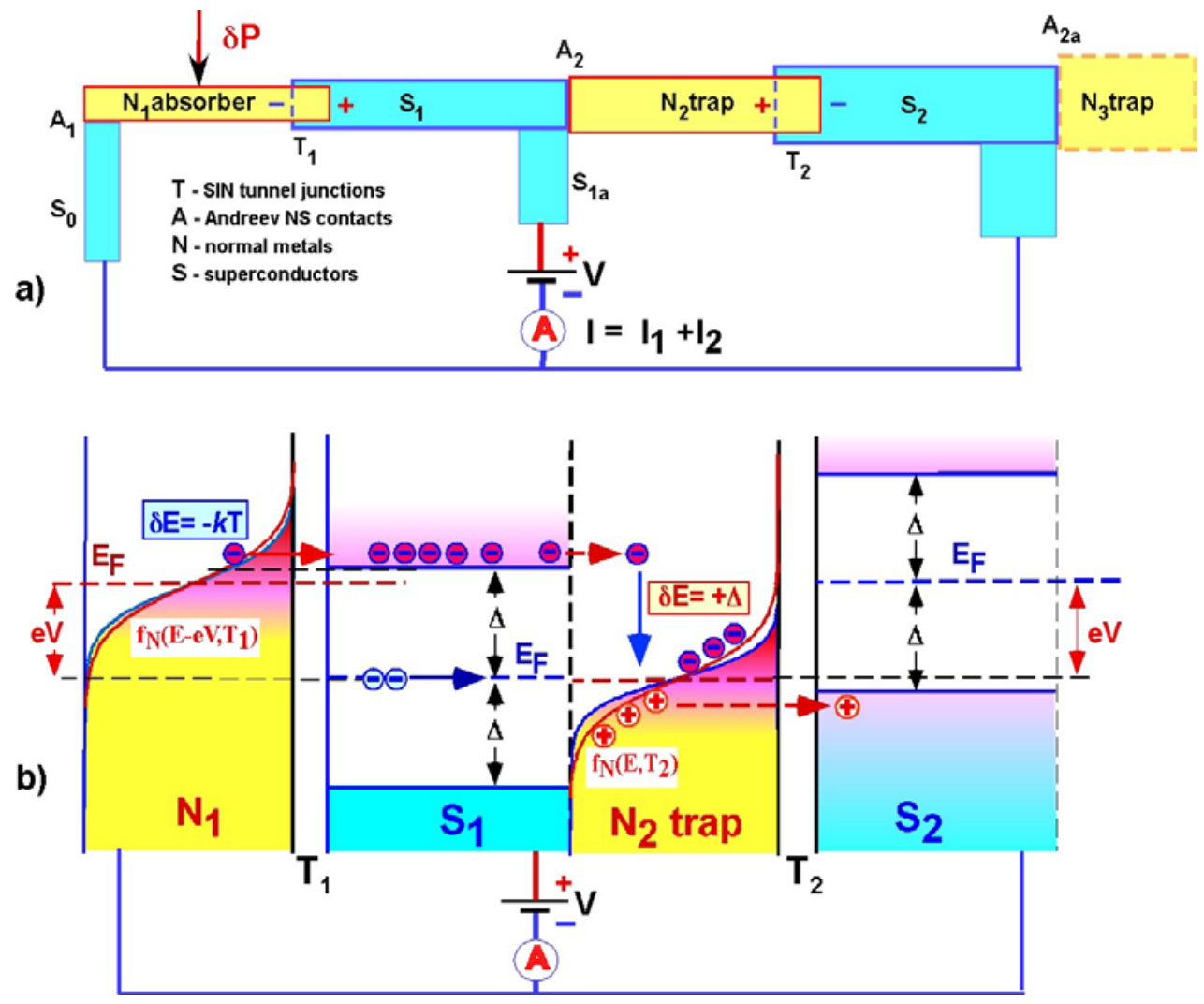

Fig. 6. Schematics of the cascade amplifier for the hot-electron microbolometer (NHEB) with trapping hot quasiparticles after tunnel junction $T_{1}$ and adding a stimulated current $I_{2}$ to the main measurement current $I_{1}$ for amplification. Note that junctions $\mathrm{T}_{1}$ and $\mathrm{T}_{2}$ (of different area) work in opposite directions with the same voltage near $\Delta$ that can be realized only in a voltage-biased mode. Trap $\mathrm{N}_{2}$ can bring amplification of the current only in regime of effective electron cooling.

The only decision we can see is using the second stage in regime of strong direct electron cooling when all released energy will be removed from the trap $\mathrm{N}_{2}$ by tunnel junction $\mathrm{T}_{2}$. In this case we can support temperature of $\mathrm{N}_{2}$ near the basic level keeping high coefficient of amplification. 
Schematics of the cascade amplifier for the hot-electron microbolometer (NHEB) with trapping hot quasiparticles after tunnel junction $\mathrm{T}_{1}$ is shown in Fig. 4a. The interesting feature of the proposed scheme is adding the stimulated current $\mathrm{I}_{2}$ to the main measurement current $\mathrm{I}_{1}$ for amplification. In this case, the coefficient of amplification will be more than unity for any regime of amplification. Note that junctions $\mathrm{T}_{1}$ and $\mathrm{T}_{2}$ (of different area) work in opposite directions with the same voltage near $\Delta$ that can be realized only in voltage-biased mode. The junction $T_{1}$ removes hot electrons from $\mathrm{N}_{1}$ but the junction $\mathrm{T}_{2}$ removes hot holes from $\mathrm{N}_{2}$ giving increase of common measuring current in both cases.

Principle of cascade amplification can be extended further for third stage (and so on) with the same adding amplified currents from junctions of increased area, biased in opposite directions by common voltage.

Numerical analysis gives the main characteristic of the cascade amplifier with one stage of amplification shown in Fig. 5. Coefficient of amplification for small signal

$K_{2}=\frac{\Delta I_{2}}{\Delta I_{1}}$

in dependence on voltage has been simulated for various values of resistance $\mathrm{R}_{2}$ of second tunnel junction. The volumes of normal metals $\Lambda_{1}$ and $\Lambda_{2}$ are the same. It is clear that effective amplification is started at the level of $R_{2}$ an order of magnitude less than $R_{1}$ (more effective electron cooling of $N_{2}$ for increased level of power in comparison with $\mathrm{N}_{1}$ ).

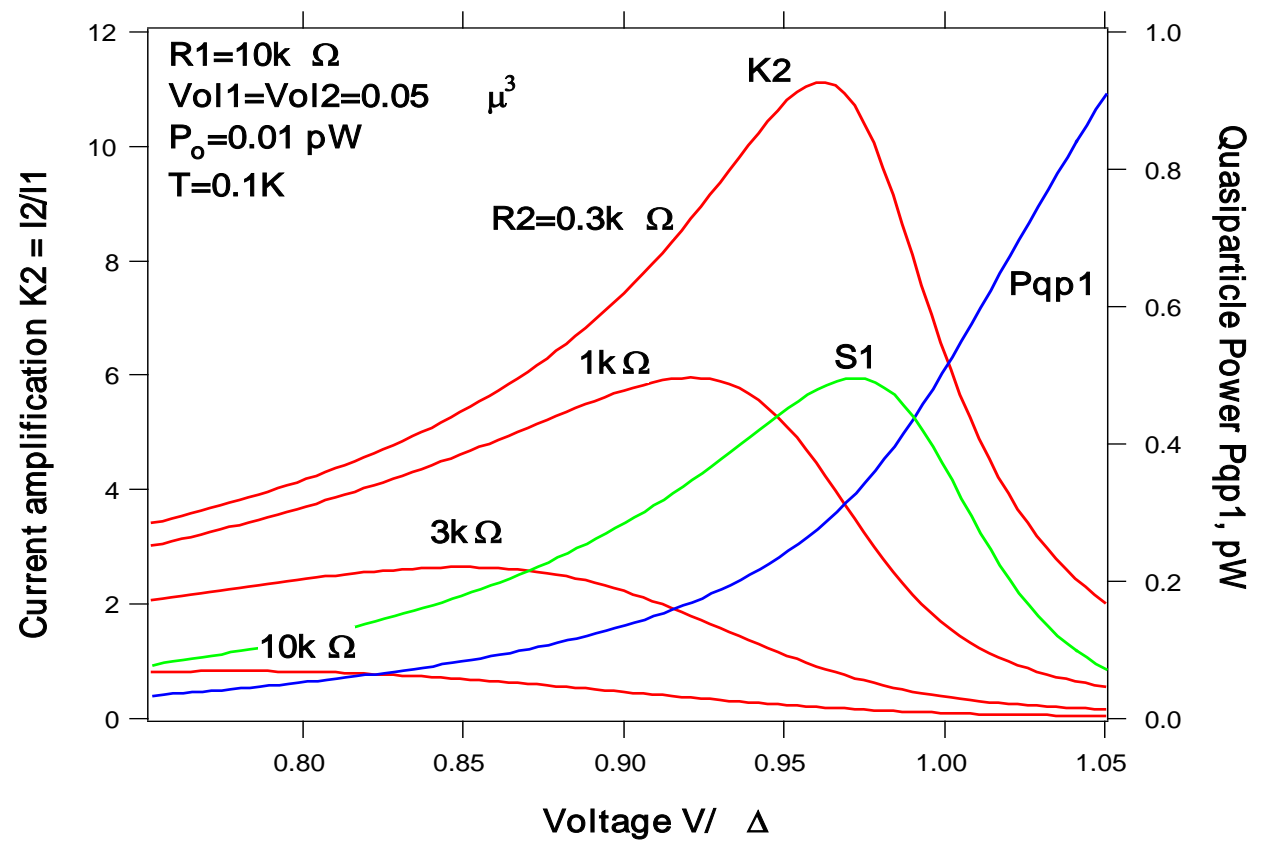

Fig. 7. Coefficient of amplification $\mathrm{K}_{2}=\Delta \mathrm{I}_{2} / \Delta \mathrm{I}_{1}$ of the cascade amplifier in dependence on voltage for various resistances of second tunnel junction $\mathrm{R}_{2}$. Responsivity of the first tunnel junction is shown by dash-dotted line and transferred power of hot quasiparticles to $\mathrm{N}_{2}$ trap, $\mathrm{P}_{\mathrm{qp} 1}$ - by dashed line. The effective amplification is started at the level of $R_{2}$ an order of magnitude less than $R_{1}$ (more effective electron cooling of $N_{2}$ for increased level of power in comparison with $\mathrm{N}_{1}$ ).

Influence of effective electron cooling on performance of amplifier can be clear seen in dependence of amplification on resistance $R_{2}$ of the second tunnel junction. If we accept the same $R_{2}$ as $R_{1}$ no amplification can be realized. It's interesting that responsivity of the first stage is reasonably good for 
the same parameters. The reason is that the first stage is under influence of the power $\mathrm{P}_{0}$ and second stage - under increased power (roughly proportionally $\Delta / \mathrm{kT}$ ). The dependence of power of hot quasiparticles $\mathrm{P}_{\mathrm{qp} 1}$ transferred to trap $\mathrm{N}_{2}$ is shown in Fig. 5 by dashed line. If we decrease $\mathrm{R}_{2}$, the effect of electron cooling is increased and values of $K_{2}$ more than 10 are realized for $R_{2} / R_{1}=30$.

To continue this line of cascade amplification with adding current we should add the same cascade to the trap $\mathrm{N}_{3}$ with the same relation of the resistors $\mathrm{R}_{3} / \mathrm{R}_{2}=10-30$.

Realization of this cascade amplifier does not need any special technology and the amplifier can be fabricated in the same vacuum circle as the main bolometer on the same chip. Cascade principle with the same voltage bias supply for all junctions in opposite polarity gives opportunity to realize amplifier without any additional power supply.

For realization of the multi-pixel array of the sensors the most important question is a number of additional wires for amplifiers. For this cascade amplifier the number of additional wires is equal zero! So, this amplifier can be very attractive for large imaging arrays of sensors [14].

\section{REFERENCES}

1. http://fy.chalmers.se/ f4agro/Submillimetron/ - Project Submillimetron

2. Gromov, V. D., L. S. Kuzmin, D. Chouvaev, L. A. Gorshkov, N.S.Kardashev, V. I. Slysh, S. F. Stoiko, M. A. Tarasov, A. G. Trubnikov, A. N. Vystavkin. "Submillimeter Telescope for the Russian Segment of the ISS: Submillimetron Project", Proceedings of "The Promise of FIRST" symposium, December 2000, Toledo, Spain, ed. G.L. Pilbratt, J. Cernicharo, A.M. Heras, T. Prusti, \& R. Harris, ESA SP-460 (2001)

3. L. Kuzmin . "Capacitively Coupled Hot Electron Microbolometer as Perspective IR and Sub-mm Wave Sensor", Proceeding of the 9th International Symposium on Space Terahertz Technology, Pasadena, pp 99-103, March 1998.

4. L. S. Kuzmin. On the concept of a hot-electron microbolometer with capacitive coupling to the antenna, Physica B: Condensed Matter, 284-288, 2129 (2000).

5. M. Nahum, P. Richards and C. Mears. IEEE Trans.on Appl. Superc., 3, 2124 (1993).

6. M. Nahum, J.M. Martinis, Appl. Phys. Lett., v. 63, N 22, pp. 3075-3077 (1993).

7. L. Kuzmin, D. Chouvaev, M. Tarasov, P. Sundquist, M. Willander, T. Claeson. IEEE Trans. Appl. Supercond., 9, pp. 31863189 (1999).

8. L. Kuzmin, I. Devyatov, and D. Golubev. "Cold-electron” bolometer with electronic microrefrigeration and the general noise analysis”. Proceeding of SPIE, v. 3465, The 4th International conference on mm and submm waves, San-Diego, pp. 193-199, July 1998.

9. D. Golubev, and L. Kuzmin, Nonequilibrium theory of a hot-electron microbolometer with NIS tunnel junction”, Journal of Applied Physics, 89, 6464-6472 (2001).

10. A. Lee, P. Richards, S. Nam, B, Cabrera, K. Irwin, A superconducting bolometer with strong electrothermal feedback, Applied Physics Letters, 69, 1801-1803 (1996).

11. J. Pekola, D. Anghel, T. Suppola, J. Suoknuuti, and A. Manninen, Trapping of quasiparticles of a nonequilibrium superconductor, Applied Physics Letters, 76, 2782-2784 (2000).

12. N. Booth, Quaiparticle trapping and quasiparticle multiplier, Applied Physics Letters, 50, 293-295 (1987).

13. N. Booth, P. Fisher, M. Nahum, and J. Ullom, A superconducting transistor based on quasiparticle trapping

14. A. Bitterman. "Superconducting Detectors for Astronomy", Superconductor \& Cryoelectronics, Vol. 12, No. 2, p17 (1999). 